\title{
Characterization of Japanese Apricot (Prunus mume) Floral Bud Development Using a Modified BBCH Scale and Analysis of the Relationship between BBCH Stages and Floral Primordium Development and the Dormancy Phase Transition
}

\author{
Tzu-Fan Hsiang, Yuan-Jui Lin, Hisayo Yamane *(D) and Ryutaro Tao
}

check for updates

Citation: Hsiang, T.-F.; Lin, Y.-J.; Yamane, H.; Tao, R. Characterization of Japanese Apricot (Prunus mume) Floral Bud Development Using a Modified BBCH Scale and Analysis of the Relationship between $\mathrm{BBCH}$ Stages and Floral Primordium Development and the Dormancy Phase Transition. Horticulturae 2021, 7, 142. https://doi.org/10.3390/ horticulturae7060142

Academic Editors: Douglas D. Archbold and Luigi De Bellis

Received: 15 April 2021

Accepted: 4 June 2021

Published: 8 June 2021

Publisher's Note: MDPI stays neutral with regard to jurisdictional claims in published maps and institutional affiliations.

Copyright: (c) 2021 by the authors. Licensee MDPI, Basel, Switzerland. This article is an open access article distributed under the terms and conditions of the Creative Commons Attribution (CC BY) license (https:// creativecommons.org/licenses/by/ $4.0 /)$.
Graduate School of Agriculture, Kyoto University, Kyoto 606-8502, Japan; hsiang.tzufan.83e@st.kyoto-u.ac.jp (T.-F.H.); lin.yuanjui.75f@st.kyoto-u.ac.jp (Y.-J.L.); tao.ryutaro.8c@kyoto-u.ac.jp (R.T.)

* Correspondence: yamane.hisayo.6n@kyoto-u.ac.jp; Tel.: +81-75-753-6052

Abstract: Bud dormancy is an important developmental stage that ensures that trees can tolerate environmental stresses in winter and bloom uniformly in the following spring. Regarding Rosaceae floral buds, exposure to chilling conditions promotes floral primordium development and the transition from endodormancy to ecodormancy. A subsequent period of warm conditions induces blooming. In Japanese apricot (Prunus mume), dormancy progression is accompanied by morphological changes that alter the bud appearance and internal structures. We used a modified BBCH scale and conducted microscopy analyses to elucidate the bud developmental stage of three cultivars with contrasting chilling requirements. The floral bud developmental period corresponding to BBCH stages 51-53 includes the transition from endodormancy to ecodormancy in all three cultivars. Male meiosis and microspore development occurred during this transition in high-chill cultivars, but were detected considerably later than the transition in the low-chill cultivar. A slow or suspended developmental phase was observed only for the high-chill cultivars upon completion of floral primordium organ differentiation, suggesting that chilling may be required to induce floral bud maturation and dormancy release only in high-chill cultivars. Possible relationships among $\mathrm{BBCH}$ stages, flowering-related morphological characteristics, and the dormancy phase transition in Japanese apricot are discussed.

Keywords: chilling requirement $(\mathrm{CR})$; floral bud; dormancy; microsporogenesis; relative growth rate (RGR); BBCH scale

\section{Introduction}

Exposure to low temperatures affects floral development in numerous species. A prolonged period of low temperatures is necessary in bulb species for normal floral organ development; otherwise, floral organs will be severely deformed [1]. Therefore, it is considered that the chilling treatment that promotes floral primordium development and maturation is necessary for the subsequent blooming and fruiting process [2]. Strictly speaking, bud dormancy refers to the state in which the bud meristem is unable to resume growth and development under suitable environmental conditions [3]. Lang (1987) divided fruit tree bud dormancy into endodormancy and ecodormancy. During endodormancy, bud growth ceases because of internal factors, and a particular period of low temperatures is required for buds to regain the competency to grow. In contrast, in ecodormancy, buds can resume growth, but unfavorable environmental conditions will arrest or delay active growth [4]. Buds shift from endodormancy to ecodormancy when the cultivar-dependent chilling requirement $(\mathrm{CR})$ is fulfilled. Moreover, a subsequent exposure to a certain period of warm conditions is required to release buds from ecodormancy so they may enter the full bloom stage. If the floral buds of Rosaceae species, including Japanese apricot (Prunus mume), are not sufficiently exposed to low temperatures, they will be unable to develop 
further, resulting in a relatively low blooming percentage [5]. Thus, low temperatures contribute to floral development and dormancy release.

Regarding Prunus fruit trees, although their floral buds do not exhibit high blooming competency during endodormancy, they apparently continue to develop during endodormancy and ecodormancy $[2,6]$. Sweet cherry (P. avium) and apricot (P. armeniaca) floral buds remain in a quiescence stage (i.e., rest) during dormancy until the $C R$ is fulfilled, after which they resume developing $[2,6]$. Endodormancy release and ecodormancy release are accompanied by morphological changes affecting the appearance and internal structures of buds (e.g., bud scale color and pollen development) [2,7-10]. The Biologische Bundesanstalt, Bundessortenamt and $\mathrm{CHemical}$ industry $(\mathrm{BBCH})$ scale systematically defines the botanical characteristics of plant developmental stages [11]. In the genus Prunus of the family Rosaceae, researchers have used BBCH scales to characterize sweet cherry ( $P$. avium L.), apricot (P. armeniaca L.), and almond (P. dulcis L.) at different stages of growth and development $[7,12,13]$. Additionally, $\mathrm{BBCH}$ scales have been applied to assess the relationship between the sweet cherry bud appearance and dormancy status [2,14]. In sweet cherry, $\mathrm{BBCH}$ stage 5 (reproductive development) was further defined using dormancy characteristics. For example, $\mathrm{BBCH}$ stage 50 represents deep dormancy, whereas $\mathrm{BBCH}$ stage 51 involves the initiation of dormancy release $[2,7,14]$. However, the relationship between the $\mathrm{BBCH}$ scale and the dormancy status remains to be elucidated in other Prunus species.

Among the histological changes during floral bud development, microsporogenesis is an important event associated with the dormancy status of Prunus species $[2,6,14,15]$. The timing of microsporogenesis is reportedly correlated with different CRs and blooming dates among apricot (P. armeniaca) and sweet cherry (P. avium) cultivars $[2,14,15]$. In apricot, after the CR of the floral buds has been fulfilled, the pollen mother cells are reactivated, which coincides with $\mathrm{BBCH}$ stage $53[6,15]$. In sweet cherry, however, male meiosis is induced after exposure to warm conditions following CR fulfillment [16].

The objective of this study was to evaluate the potential relationship between morphological characteristics and the dormancy phase transition in Japanese apricot floral buds. Accordingly, we determined the timing of the transition from endodormancy to ecodormancy in three cultivars with contrasting blooming dates. To clarify floral bud growth characteristics, relative growth rate (RGR) and water content were investigated. Previous studies revealed a relationship between water content trends and dormancy progression in several Rosaceae fruit trees [2,17]. We also described Japanese apricot floral bud's phenological stages using a modified $\mathrm{BBCH}$ scale for the first time and observed the internal development of floral organs. Here, we discuss the relationship between morphological development and dormancy phase transition in Prunus fruit trees.

\section{Materials and Methods}

\subsection{Plant Materials}

Adult trees (>15 years old) of 19 Japanese apricot cultivars grown at the Kyoto farmstead of the experimental farm of the Graduate School of Agriculture, Kyoto University (35.032 N, 135.785 E), Kyoto, Japan, were used in this study. We determined the blooming date (BD) of each cultivar in the 2019-2020 season. BD was defined as the date when $50 \%$ of the floral buds on a tree were in the original BBCH stage 57 [11], with visible petal tips in the field. The temperature was recorded hourly using the T\&D Thermo Recorder, TR-50U2 (T\&D Corporation, Matsumoto, Japan).

\subsection{Evaluation of Growth/Dormancy Status}

To investigate the timing of the dormancy phase transition in the 2019-2020 season, bud break competency was tested as described by Fadón et al. [18]. Branches were collected on the 25th of each month from three cultivars with contrasting blooming dates ('Nanko', 'Shirokaga', and 'Ellching') and separated into several pieces (single-node cuttings), and a total of five replicates and 15 pieces from each replicate were used. Then, single-node cuttings were incubated under forcing conditions $\left(18 \mathrm{~h}\right.$ day $/ 6 \mathrm{~h}$ night, $\left.23^{\circ} \mathrm{C}\right)$. The bud 
break rate was recorded twice weekly until 3 weeks after initiating the incubation. In the 2020-2021 season, bud break competency was tested in 'Nanko' using branches collected on 30 November and 31 December. We also calculated the RGR and water content to compare seasonal bud growth patterns among the three cultivars. A total of four replicates were used in the RGR calculation, and each replicate included 20 buds. The RGR was calculated using the following formula:

$$
\mathrm{RGR}=\frac{\mathrm{W} 2-\mathrm{W} 1}{(\mathrm{~T} 2-\mathrm{T} 1) \cdot \mathrm{W} 1}
$$

where $\mathrm{W} 1$ is bud fresh weight at time point $1(\mathrm{~g}), \mathrm{W} 2$ is bud fresh weight at time point $2(\mathrm{~g})$, $\mathrm{T} 1$ is time point 1 (day), and T2 is time point 2 (day).

The collected buds were lyophilized and weighed. A total of four replicates were used in water content (\%) calculation, and each replicate included 20 buds. The water content was calculated using the following formula:

Water content $(\%)=($ fresh weight - dry weight $) /$ fresh weight $\times 100$.

\subsection{Estimation of the CR for Endodormancy Release and the Heat Requirement (HR) for Blooming}

The date of transition from endodormancy to ecodormancy (i.e., the date of CR fulfillment) was defined as the date when the collected branches had a $50 \%$ bud break rate under forcing conditions, which was estimated by a linear regression of increasing seasonal bud break competency determined using the above-described method. We used the following three models for evaluating the CR: (1) chilling hour model [19], (2) Utah model [20], and (3) dynamic model [21]. Regarding the chilling hour model, all hours with temperatures between 0 and $7.2{ }^{\circ} \mathrm{C}$ were considered chilling hours [19]. For the Utah model, the chill unit (CU) for hourly temperatures was calculated using the conversion table of Utah CUs [20]. The dynamic model was applied using a program available on the University of California Division of Agriculture and Resources website (https: / / ucanr. edu/sites/fruittree/How-to_Guides/Dynamic_Model_-_Chill_Accumulation/).

The growing degree hour $(\mathrm{GDH})$ value was assigned for all hours with temperatures between 4.5 and $25^{\circ} \mathrm{C}$, and was calculated as the temperature minus $4.5^{\circ} \mathrm{C}$. The HR was calculated as the accumulated GDH values from the CR fulfillment date to the BD [22,23].

\subsection{Analyses of Bud Phenology and Internal Floral Primordium Development}

The floral buds of the three cultivars grown in the field were photographed twice monthly (on average) from September to February in the 2019-2020 season. The 'Nanko' floral buds were also photographed weekly in December during the 2020-2021 season. Floral bud samples were collected every 2 weeks in the 2019-2020 season (28 September, 14 October, 29 October, 15 November, 30 November, 15 December, and 30 December) and were preserved in FAA fixative solution (formaldehyde/alcohol/acetic acid/ $\mathrm{H}_{2} \mathrm{O}=2: 10: 1: 7$ ). After replacing the fixative solution with a series of sucrose solutions $(10 \%, 20 \%$, and $30 \%$ ) for $2 \mathrm{~h}$ each, the samples were embedded in Super Cryoembedding Medium (Leica Microsystems $\mathrm{GmbH}$, Wetzlar, Germany) and then frozen using isopentane as the refrigerant in liquid nitrogen. For each sample, 7-15 $\mu \mathrm{m}$ longitudinal sections were prepared using a cryostat microtome (CM1520, Leica) and stained with toluidine blue O solution (10 $\mathrm{mM}$ benzoic acid, $10 \mathrm{mM}$ sodium benzoate, and $2 \mathrm{mM}$ toluidine blue $\mathrm{O}$ ). The floral bud sections were examined using the DP80 light microscope (Olympus, Tokyo, Japan) and photographed.

\section{Results}

\subsection{Contrasting the $C R$ and HR of Three Cultivars}

On the basis of the distribution of the BD, 19 cultivars from the experimental farm of Kyoto University were classified as early blooming, medium blooming, late blooming, and extra late blooming (Figure 1; Table 1). In this study, one representative cultivar was 
selected from each blooming date category, with the exception of medium blooming, and used for the analyses of morphology, organ development, and dormancy status. The BD of 'Ellching' was 6 January 2020, whereas it was 4 and 10 February 2020 for 'Nanko' and 'Shirokaga', respectively.

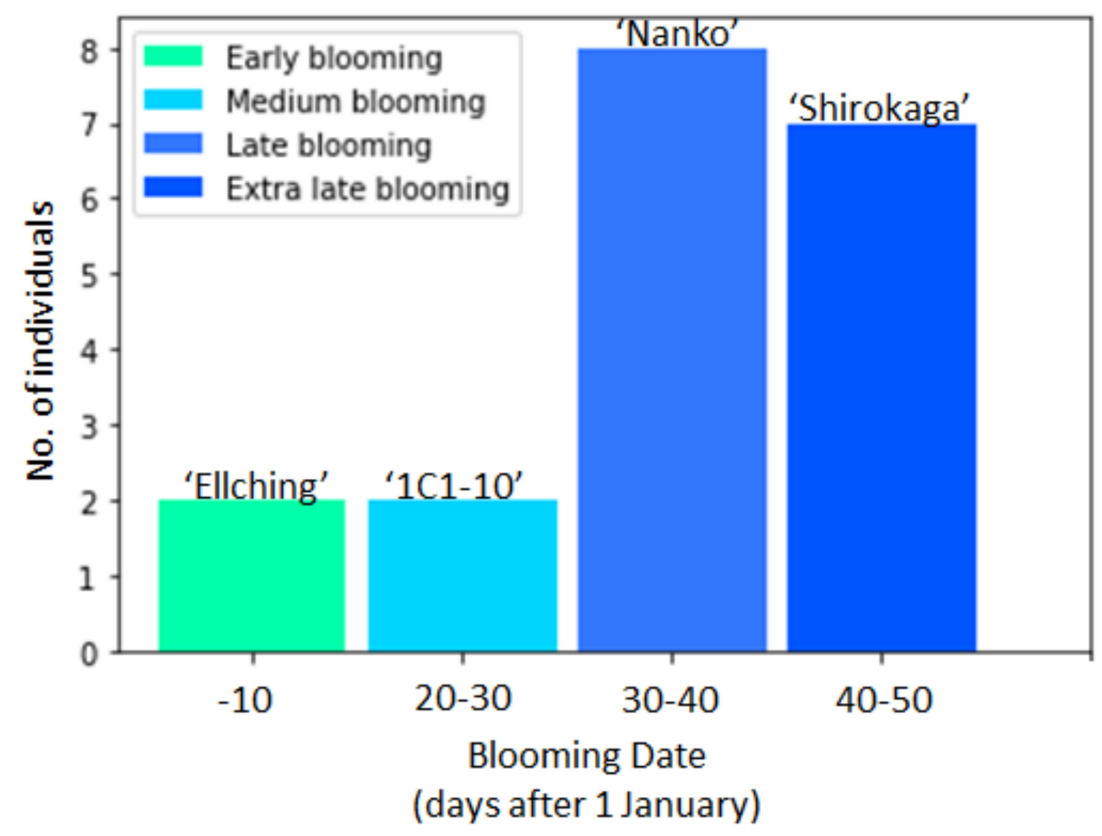

Figure 1. Frequency distribution of blooming dates in the 19 Japanese apricot collections during the 2019-2020 season. 'Ellching', early blooming; '1C1-10', medium blooming; 'Nanko', late blooming; 'Shirokaga', extra late blooming.

Table 1. Blooming date (BD) for 19 Japanese apricot cultivars in the 2019-2020 season.

\begin{tabular}{ccc}
\hline Cultivar & BD2020 & Origin \\
\hline Ellching & 6 & Taiwan \\
Hayazakinanko & 7 & Japan \\
1C1-10 & 24 & Japan \\
Kairyo-uchida & 28 & Japan \\
Ryukyokoume & 32 & Japan \\
1KO-26 & 33 & Japan \\
Benisashi & 34 & Japan \\
Nanko & 35 & Japan \\
Kotsubnu-nanko & 35 & Japan \\
Koshinoume & 35 & Japan \\
Kagajizo & 37 & Japan \\
Kensaki & 38 & Japan \\
Shirokaga & 41 & Japan \\
Hachiro & 47 & Japan \\
Rinshu & 49 & Japan \\
Koshukoume & 49 & Japan \\
Aojiku & 49 & Japan \\
Oshuku & 49 & Japan \\
Gyokuei & 49 & Japan \\
\hline
\end{tabular}

${ }^{1}$ BD2020: blooming date was calculated from 1 January 2020 (Julian days).

On the basis of the seasonal changes in bud break competency, the CR fulfillment dates were estimated to be 12 November, 29 December, and 8 January for the earlyblooming 'Ellching', late-blooming 'Nanko', and extra-late-blooming 'Shirokaga', respectively (Figure 2). Using the chilling hour model (indicated by $\mathrm{CH}$ ) [19], the Utah model 
(indicated by CU) [20], and the dynamic model (indicated by CP) [21], the CRs were calculated as follows: 'Ellching', 2 CH, 5.5 CU, and 2 CP; 'Nanko', $395 \mathrm{CH}, 584.5 \mathrm{CU}$, and 31.5 CP; and 'Shirokaga', $519 \mathrm{CH}, 754.5 \mathrm{CU}$, and 39.3 CP. The starting point of chilling temperature accumulation was estimated from 9 November, 8 November, and 6 November for the chilling hour model, the Utah model, and the dynamic model, respectively. The results of the three models reflect the differences in the early and late blooming dates (i.e., the extra-late-blooming cultivar had the highest CR) (Figure 3). Therefore, we defined 'Ellching', 'Nanko', and 'Shirokaga' as low-, high-, and extra-high-chill cultivars, respectively. The analysis using the GDH model revealed that 'Ellching' and 'Shirokaga' had the highest (6294.4) and lowest (2436.8) GDH values, respectively (Figure 4).

\section{Endodormancy Ecodormancy}

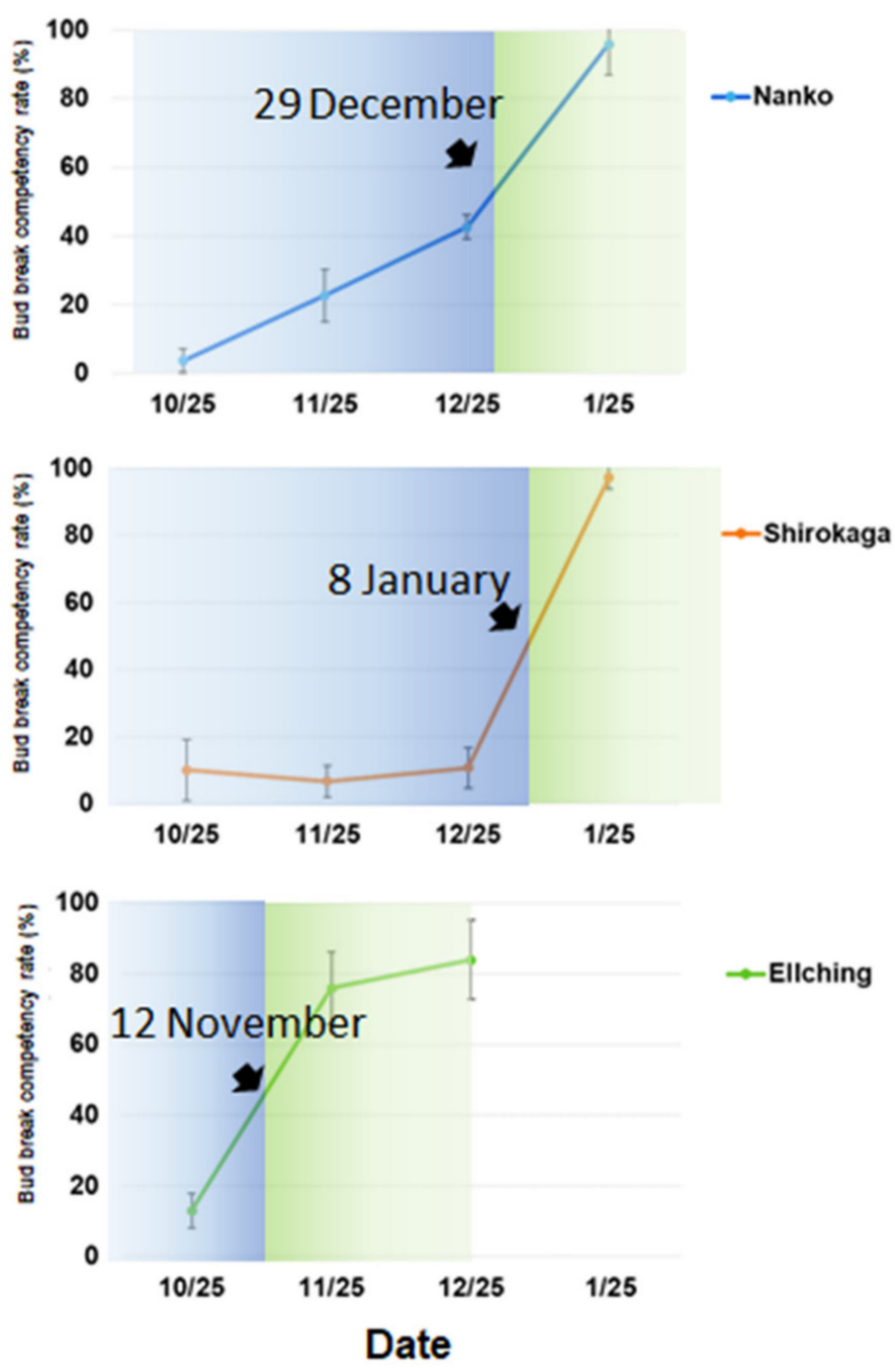

Figure 2. Seasonal changes in the bud break competency of the floral buds of three cultivars. The CR fulfillment dates were estimated to be 29 December, 8 January, and 12 November for the late-blooming 'Nanko', the extra-late-blooming 'Shirokaga', and the early-blooming 'Ellching', respectively. Five replicates were analyzed, and 15 nodes were examined for each replicate. The error bars represent the SE. 


\section{Chilling hour model}
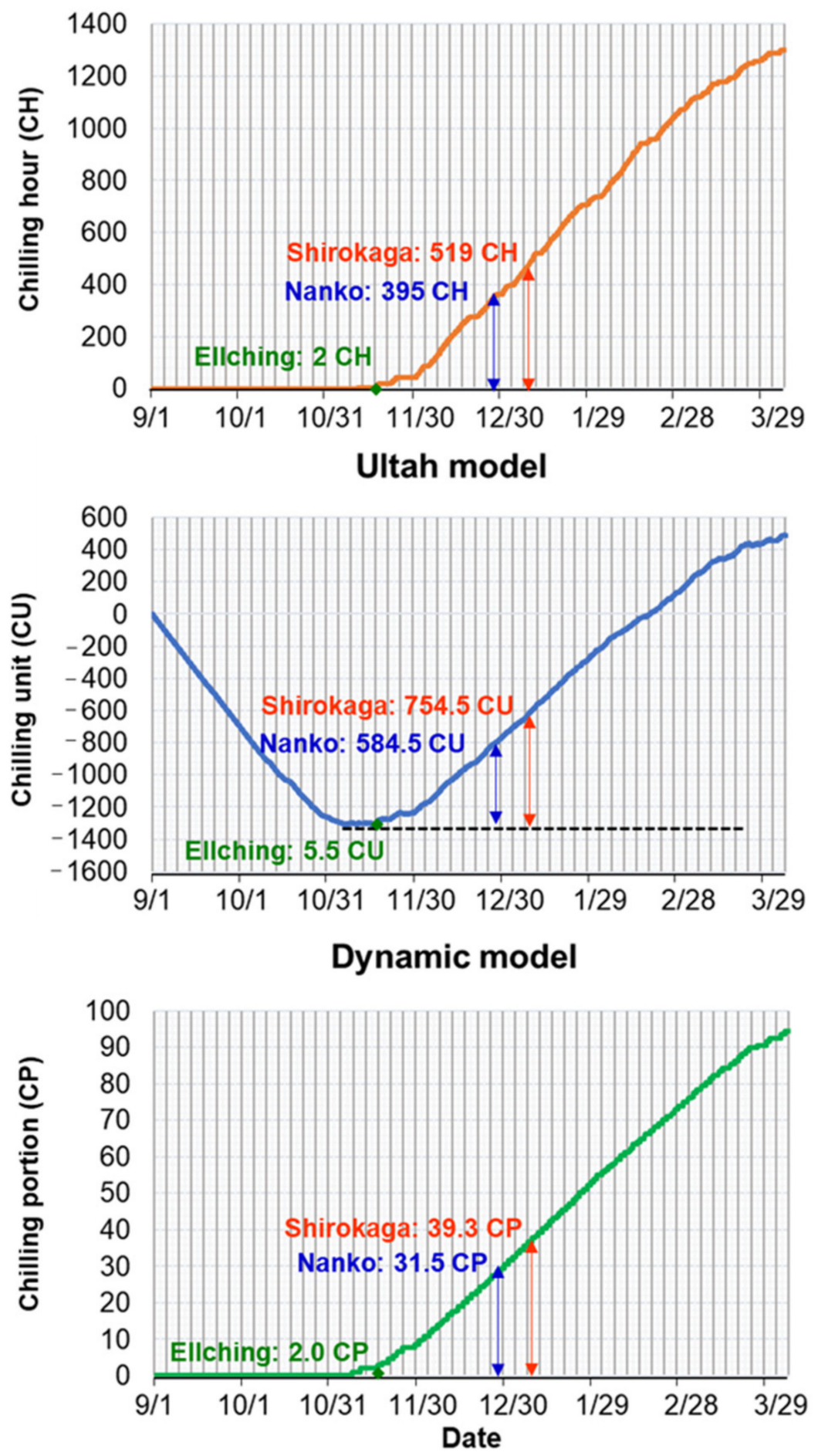

Figure 3. Estimation of the CR of three cultivars using the chilling hour model (top), the chill unit (Utah) model (middle), and the dynamic model (bottom). 'Nanko', high chill; 'Shirokaga', extra high chill; 'Ellching', low chill. 


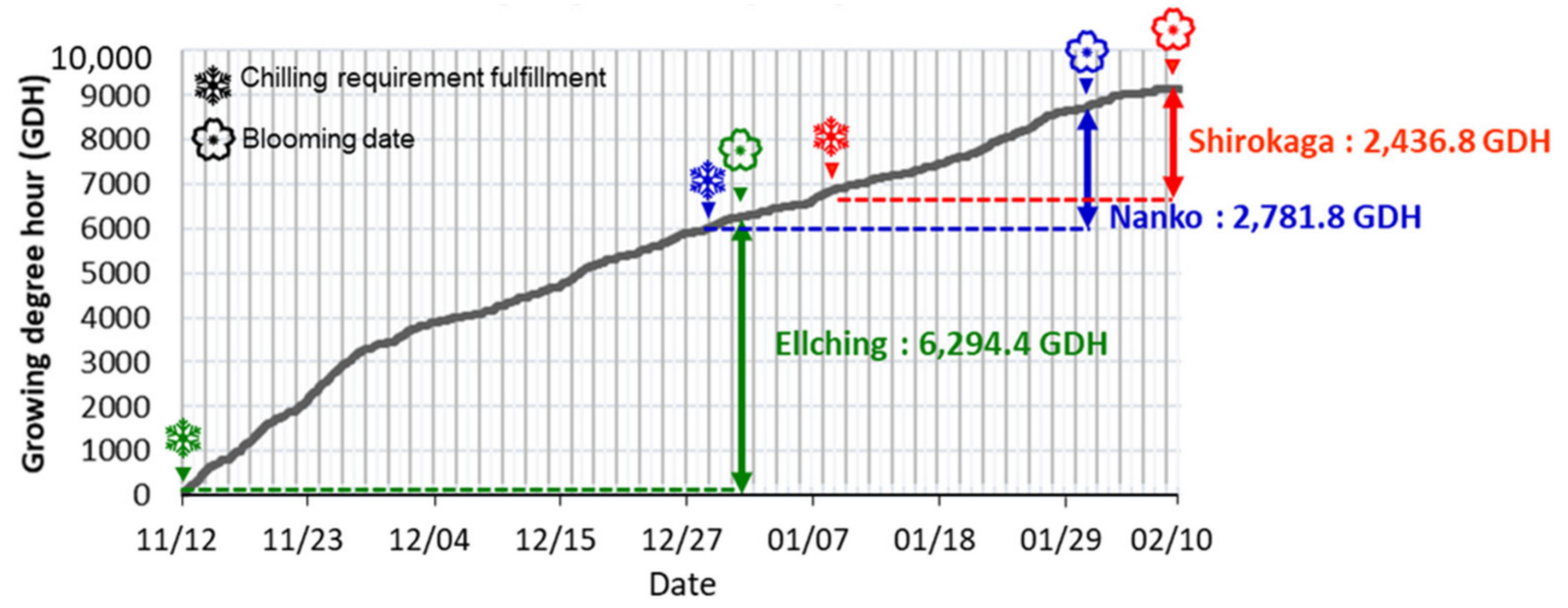

Figure 4. Evaluation of the HR of three cultivars using the growing degree hour (GDH) model. 'Nanko', high chill; 'Shirokaga', extra high chill; 'Ellching', low chill. Ice crystal mark means chilling requirement fulfillment; flower mark means blooming date.

\subsection{Seasonal Changes in the RGR and Water Content of the Low-Chill and High-Chill Cultivars}

An examination of the floral bud development of all tested cultivars revealed a low RGR from September to October (Figure 5a). The low-chill cultivar ('Ellching') grew rapidly $\left(0.14 \mathrm{~g} \cdot \mathrm{g}^{-1} \cdot \mathrm{day}^{-1}\right)$ from November to December. In contrast, the high-chill and extra-highchill cultivars had a relatively stable RGR $\left(0.05-0.08 \mathrm{~g} \cdot \mathrm{g}^{-1} \cdot \mathrm{day}^{-1}\right)$ from November to January. The floral bud water content increased consistently during dormancy progression (from $30 \%$ to $80 \%$ ) (Figure 5 b), with a similar trend detected for all three cultivars.

(a)

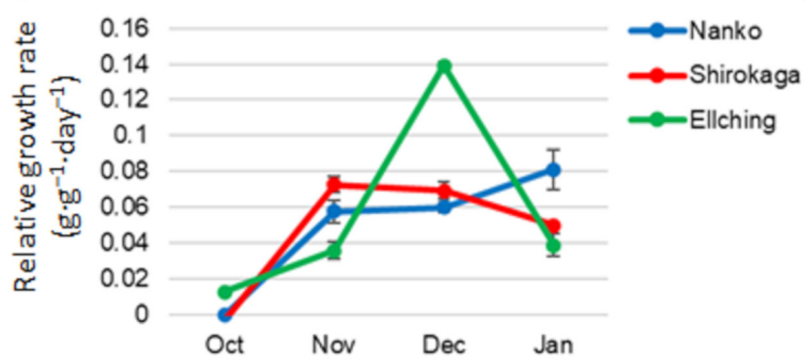

(b)

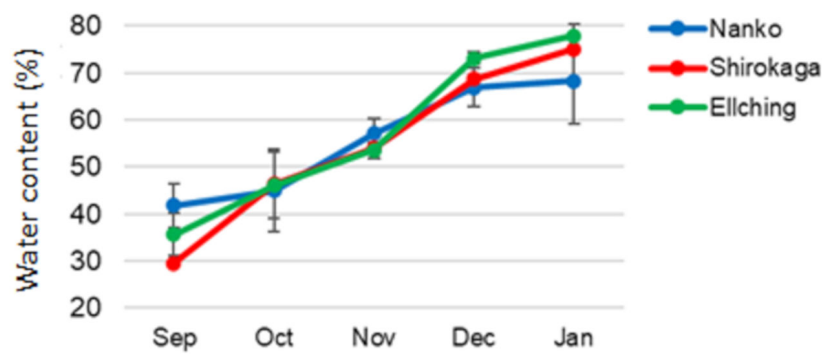

Figure 5. Seasonal changes in the relative growth rate and the water content during dormancy in 2019-2020. (a) Relative growth rate. (b) Water content (\%). 'Nanko', high chill; 'Shirokaga', extra high chill; 'Ellching', low chill. Four replicates were analyzed, and 20 flower buds were examined for each replicate. The error bars represent the SE.

\subsection{Establishment of a Modified BBCH Scale for Japanese Apricot Floral Buds}

To establish a BBCH scale for Japanese apricot floral buds, we modified the original $\mathrm{BBCH}$ scale [11]. More specifically, because Japanese apricot does not form inflorescences, we omitted the description of stage 55 (single flower buds visible (still closed) borne on short stalks) from the $\mathrm{BBCH}$ scale. In the modified $\mathrm{BBCH}$ scale, 'principal growth stage 5 : flower emergence' was used to further describe the floral bud development from dormancy to blooming in the three tested cultivars (Figure 6). Both 'Nanko' and 'Shirokaga' remained in BBCH stage 50 from September to November (Figure 6c), whereas 'Ellching' transitioned into stages 51-53 in late October (Figure 6c). In December, the 'Nanko' and 'Shirokaga' floral buds were in $\mathrm{BBCH}$ stages 51-53. In contrast, most 'Ellching' floral buds were in BBCH stages 54-56. Additionally, the 'Nanko' floral bud appearance underwent similar changes in BBCH stages 51-53 in December during the 2019-2020 and 2020-2021 seasons 
(Figure 7). Most 'Nanko' and 'Shirokaga' floral buds were in BBCH stages 54-56 until early January when they transitioned into $\mathrm{BBCH}$ stages $57-59$ (i.e., blooming period). The 'Ellching' floral buds remained in BBCH stages 54-56 for a longer period from December to early January, after which they entered the blooming period.

(a)

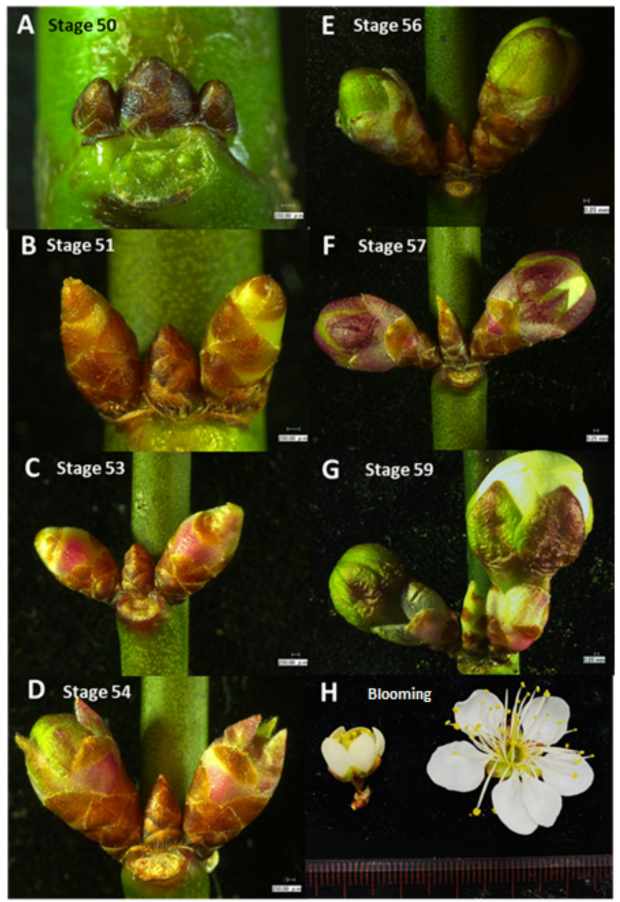

(b)
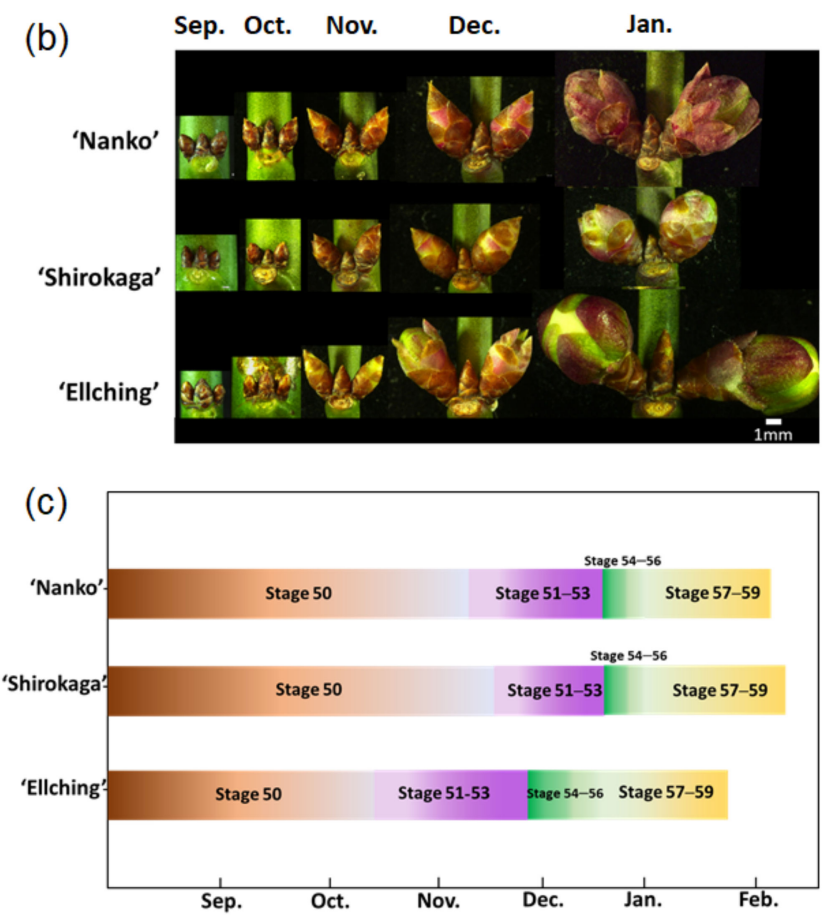

Figure 6. Evaluation of three Japanese apricot cultivars during dormancy using the modified BBCH scale. (a) Floral bud developmental stage. Scale bar $=250 \mu \mathrm{m}$. (b) Seasonal changes in the bud appearance among Japanese apricot cultivars with varying blooming dates. Scale bar $=1 \mathrm{~mm}$. (c) $\mathrm{BBCH}$ scheme of three Japanese apricot cultivars during dormancy. 'Nanko', high chill; 'Shirokaga', extra high chill; 'Ellching', low chill.
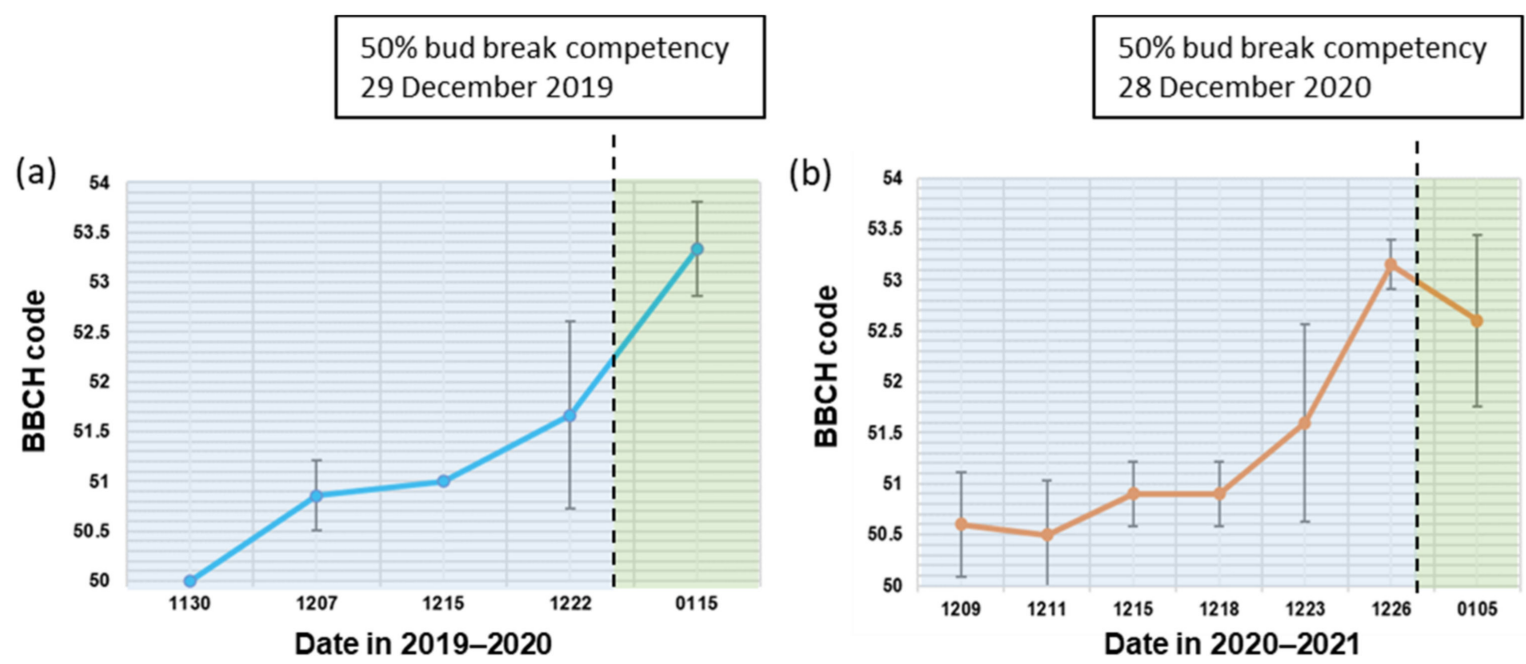

Figure 7. BBCH scale evaluation of 'Nanko' floral buds during December in 2019-2020 and 2020-2021 seasons: (a) 2019-2020, (b) 2020-2021. Ten flower buds from each stage were analyzed in 2019/2020 and 20 flower buds were examined from each stage. The error bars represent the SE. Dash line means the time point of $50 \%$ bud break competency. 


\subsection{Comparison of Seasonal Floral Primordium Development among Cultivars with Contrasting CRs}

The floral initiation (initiation of floral organ formation) of the low-chill cultivar ('Ellching') was induced relatively late, but the floral organs rapidly formed by late October (Figure 8a). The floral primordium developed earlier in the high-chill cultivars than in 'Ellching', and pistil formation was completed by the end of October. The timing of pollen maturation differed between 'Ellching' and the high- and extra-high-chill cultivars (Figure 8b). Degraded tapeta and mature pollen were detected in anthers on 15 December for 'Ellching', but on 30 December for 'Nanko' and 'Shirokaga'.
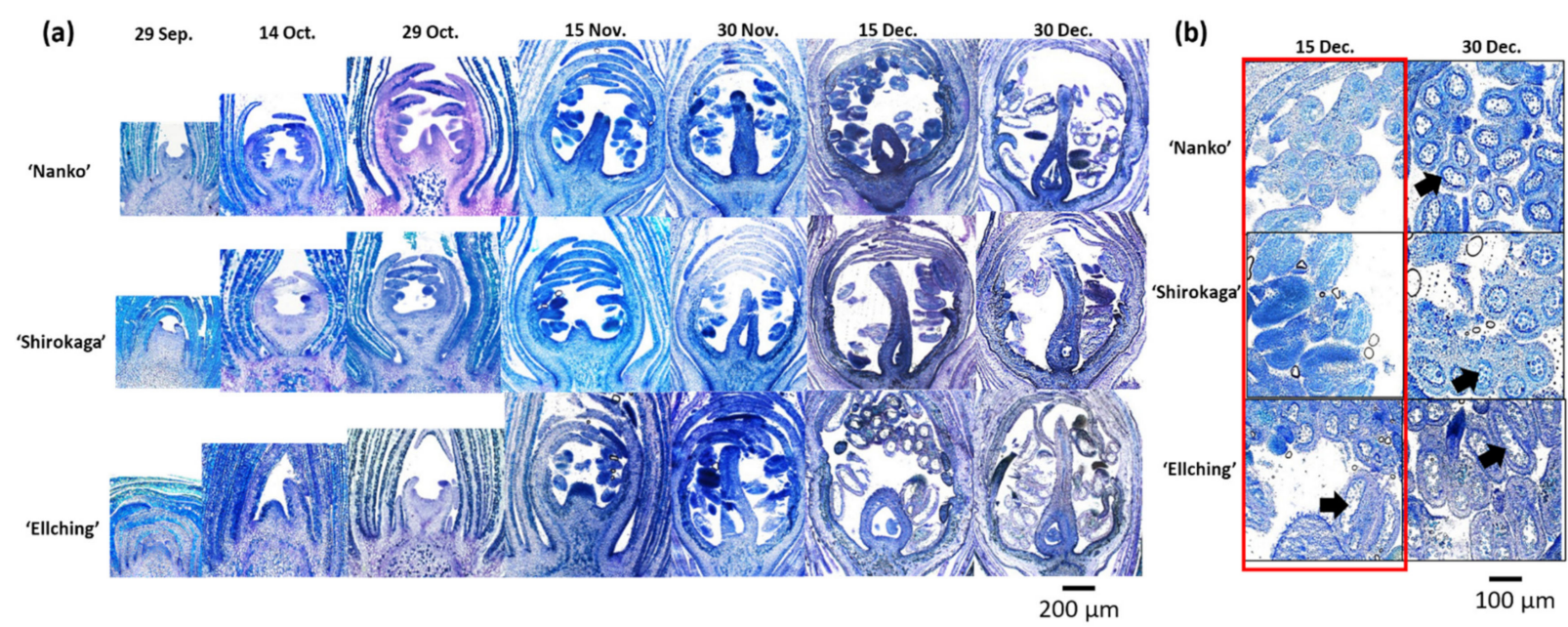

Figure 8. Longitudinal sections of the floral buds from different Japanese apricot cultivars during dormancy. (a) Floral organ: A, anther; C, carpel; P, petals; O, ovary. Scale bar $=200 \mu \mathrm{m}$. (b) Differences in pollen developmental status among cultivars. Scale bar $=100 \mu \mathrm{m}$. 'Nanko', high chill; 'Shirokaga', extra high chill; 'Ellching', low chill.

\subsection{Correlation between BBCH Stages and the Floral Bud Physiological Dormancy Status}

The results of a Pearson correlation analysis indicated that $\mathrm{BBCH}$ stages were highly positively correlated with bud break competency, RGR, and water content (correlation coefficients: $0.88,0.51$, and 0.82 , respectively) (Figure 9).

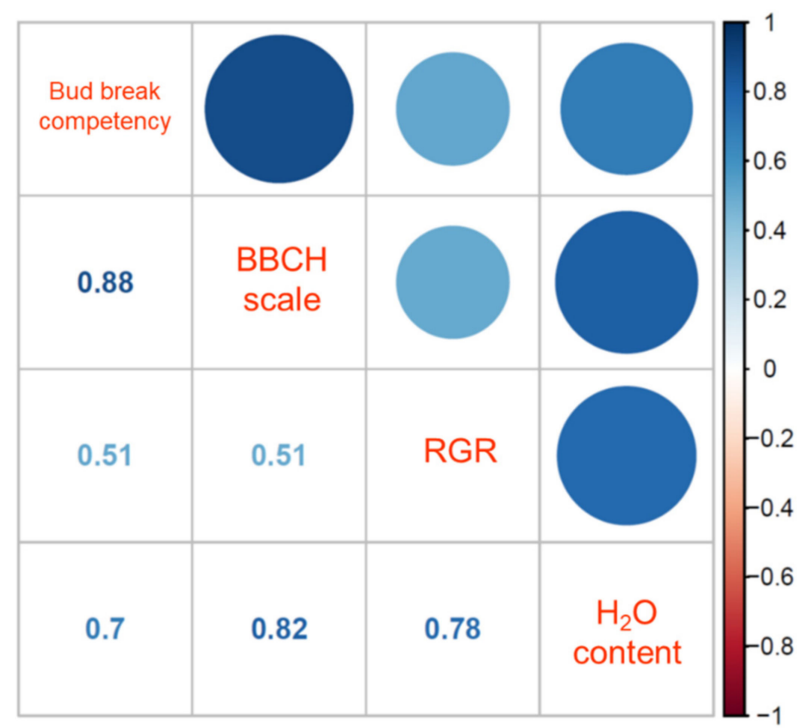

Figure 9. Pearson correlation matrix of traits during dormancy. The color scale reflects the correlation, with 1 indicating a completely positive correlation (dark blue) and -1 indicating a completely negative correlation (dark red) between two traits. 


\section{Discussion}

\subsection{Comparison of the Japanese Apricot Floral Bud Dormancy Progression of Cultivars with} Different Blooming Dates

Seasonal changes in bud break competency suggest that earlier and later endodormancy release may be primarily responsible for the earlier and later blooming of 'Ellching' and 'Shirokaga', respectively, compared with 'Nanko'. Thus, we categorized 'Ellching', 'Nanko', and 'Shirokaga' as low-chill, high-chill, and extra-high-chill cultivars, respectively. We evaluated the growth/dormancy physiological status of these cultivars by analyzing the water content and RGR. Earlier research proved that the floral bud water contents of sweet cherry [24] and Japanese pear (Pyrus pyrifolia) [25,26] increase gradually from the dormancy stage to the blooming stage. Moreover, the free water content is reportedly correlated with floral primordium development and endodormancy release in Japanese pear [25]. In the current study, the water contents of all cultivars increased until the blooming stage. However, no clear difference was observed between the low-chill and high-chill cultivars. Additionally, for all cultivars, the RGR was lowest in October. The high-chill cultivars had a constant RGR from November onward, whereas the RGR of the low-chill cultivar increased substantially in December. These results suggest that floral bud growth ceased in October, but resumed somewhat from November onward as dormancy progressed in all three cultivars. This is consistent with the results of a previous study, in which the floral development of Rosaceae fruit trees continued during dormancy [5]. Floral organ formation was observed in October and was completed by the end of October in all three cultivars. Additionally, endodormancy was released in early November, late December, and early January in the low-chill, high-chill, and extra-high-chill cultivars, respectively. Therefore, for 'Ellching', there was either no time or a very short period after floral organ formation before endodormancy release. In contrast, 'Nanko' and 'Shirokaga' had a slow or suspended developmental phase that lasted approximately 2 months before endodormancy release. We hypothesize that the $\mathrm{CR}$ of the low-chill cultivar reflects the low-temperature requirement for organ development and floral bud maturation, whereas the CRs of the high-chill cultivars reflect the low-temperature requirement for floral bud maturation and the release of the slow or suspended developmental phase.

\subsection{Calculated CRs and HRs for Japanese Apricot Floral Buds}

In this study, the CRs calculated using three different models confirmed that 'Ellching' is a low-chill cultivar and 'Nanko' is a high-chill cultivar, which is consistent with our previous findings $[27,28]$. However, the CRs indicated by $\mathrm{CH}$ determined in this study are not identical to those calculated by Yamane et al. (2006). More specifically, the CRs for 'Nanko' and 'Ellching' floral buds were approximately 500 and $300 \mathrm{CH}$, respectively, in the earlier study [27], whereas they were lower in the current study (i.e., 395 and $2 \mathrm{CH}$, respectively). The methodology for $\mathrm{CR}$ calculation was not exactly the same between these two studies, which may affect inconsistency in $C R$ values. These results also imply that the genotype-dependent CR might not be precisely determined by the chill hour model. Alternatively, even though the CR is genotype dependent and genetically controlled, it varies depending on the environmental condition and tree age. Additionally, an extremely low CR value of 'Ellching' estimated in this study may raise the question of whether "Ellching' is "low chill" or "no chill" for endodormancy release.

Regarding the HR, the GDH model indicated that 'Ellching' (low-chill cultivar) had a higher GDH value (6294) than 'Nanko' (2781) or 'Shirokaga' (2436) (Figure 4). The higher GDH value for 'Ellching' may reflect the longer ecodormancy period for this cultivar, with 56 days between the dormancy transition date and the blooming date, unlike the 36 and 33 days between these two dates for 'Nanko' and 'Shirokaga', respectively. Similar results were obtained for the HR estimated using the GDH model in apricot. Although the GDH value of the apricot cultivar 'Penta' (8923) was higher than that of 'Tardona' (7789), 'Tardona' bloomed approximately 10 days later than 'Penta' ('Penta', 12 March; 'Tardona', 21 March) [29]. Considering that a prolonged chilling period reportedly de- 
creases the HR for blooming in peach [30], a mutual compensation between chilling and warm temperature requirements for blooming may occur. This leads to the question of whether the higher GDH value for the low-chill cultivar may reflect a greater HR, namely, lower sensitivity to warm conditions, or the HR may not be accurately determined using the GDH model. Alternatively, the HR initiation date determined on the basis of bud break competency ( $>50 \%$ bud break under forcing conditions) may be inappropriate for estimating the genotype-dependent HR, especially for low-chill cultivars. Current models for estimating the dormancy status and blooming mostly focus on temperature alone. Moreover, dormancy induction is regulated by multiple environmental factors in Prunus species (e.g., the photoperiod, temperature, and water deficit stress) [31-33]. Thus, dormancy progression and release as well as blooming may be regulated by temperature and other environmental factors. Using their developmental rate model for predicting the CR and HR, Kitamura et al. (2017) concluded that warm conditions during ecodormancy may be the primary factor explaining the year-dependent blooming date of 'Nanko', rather than cold conditions during endodormancy [28]. Collectively, flowering time (blooming) models, especially for HR predictions, must be improved for future dormancy studies and for practical use.

\subsection{Relationship between the Dormancy Phase Transition and BBCH Stages in Japanese Apricot}

In this study, we established a BBCH scale applicable for Japanese apricot and compared it with the physiologically determined dormancy status. We revealed that $\mathrm{BBCH}$ stage 50 represents deep dormancy. In this stage, floral buds exhibit relatively low bud break competency, and the dormant buds are covered by dark brown scales (Figure 6). Previous investigations indicated that $\mathrm{BBCH}$ stage 50 corresponds to deep dormancy in sweet cherry [2,7]. During BBCH stage 50, floral organ primordia differentiated, and the stigma, anthers, and pistil were detectable (Figures 6 and 8). In BBCH stage 51, endodormant floral buds before CR fulfillment were swollen and covered by light brown scales (Figure 6; Table 2). In 'Nanko', bud break competency started to increase in BBCH stage 51. Additionally, BBCH stages 51-53 corresponded to the dormancy phase transition period (i.e., from endodormancy to ecodormancy), whereas BBCH stages 56-59 represented the blooming period, during which the bud break rate approached $100 \%$ under forcing conditions $(16 \mathrm{~h}$ day $/ 8 \mathrm{~h}$ night, $23^{\circ} \mathrm{C}$ ) (Figures 2 and 6). BBCH stages 51-53 corresponded to dormancy phase transition in the next growing season in 'Nanko', which supported the idea that the stages may represent dormancy transition (Figure 7). Our data indicated that the bud break competency was highly correlated with the BBCH stages (correlation coefficient of 0.88) (Figure 9), suggestive of a potential relationship between the physiological dormancy status and the apparent floral bud morphological changes in Japanese apricot.

Table 2. Description of the modified BBCH scale for Japanese apricot floral buds from dormancy to blooming.

\begin{tabular}{ll}
\hline & \multicolumn{1}{c}{ Principal Growth Stage 5: Flower Emergence } \\
\hline 50 & Floral bud closed, dark brown scales visible \\
51 & Floral bud swelling, bud still closed, light brown scales visible \\
53 & Scales further separated, light green bud sections, and light purple or pink scales visible \\
54 & Internal floral organ enclosed by light green sepals, round green sepals visible \\
56 & Flower pedicel elongating, green sepals still closed showing a round shape \\
57 & Sepals open: petal tips visible, flower with white petals (still closed) \\
59 & Flowers with petals forming a hollow ball \\
\hline
\end{tabular}

\subsection{Connection between Pollen Maturation and the Dormancy Status in Japanese Apricot}

Microsporogenesis is a biological indicator of CR fulfillment and reflects the reactivation of cells following dormancy in apricot and sweet cherry [6,8,15,34,35]. Microspore formation coincides with BBCH stage 53 in sweet cherry and apricot [2,36]. Regarding the Japanese apricot cultivars examined in this study, the timing of pollen maturation 
matched the timing of CR fulfillment in 'Nanko' and 'Shirokaga' (Figures 2 and 4), which is consistent with the findings of earlier sweet cherry and apricot studies $[6,8,15,35]$. However, for 'Ellching' (low-chill cultivar), there was approximately 1 month between CR fulfillment and pollen maturation (Figures 2 and 8). In the high-chill cultivars, tapetum degradation during the pollen maturation process was observed in $\mathrm{BBCH}$ stages 53 and 54 , and $\mathrm{BBCH}$ stages 54-56 were shorter (corresponding to a lower GDH value) than in the low-chill cultivar. In earlier investigations of apricot and sweet cherry, the plants used for analyzing pollen mother cell development were mostly high-chill cultivars, including sweet cherries 'Burlat' (981 CU) and 'Bing' (1082 CU) [2,8] and apricot 'Moniqui' (1050-1150 CU) [6]. The low-chill cultivar 'Ellching' originated in Taiwan, which is in a tropical and subtropical region [37]. Additionally, its 5.5 CU calculated using the Utah model was substantially lower than the CUs of the other Japanese apricot cultivars (Figure 3). This suggests that pollen maturation may be useful as a biomarker of endodormancy release and CR fulfillment only for high-chill cultivars in Prunus.

\section{Conclusions}

New classifications and terminologies to describe plant dormancy at the cellular level were recently proposed by Considine and Considine (2016) [38]. On the basis of the classification by Considine and Considine (2016) and the fact that bud growth continued after pistil formation until blooming in Japanese apricot, we propose that Japanese apricot floral buds may not enter a dormancy period, but become quiescent (i.e., slow or suspended development). Hence, the floral bud dormancy process of Rosaceae fruit trees may need to be redefined and reclassified in future studies. Additionally, our results suggest that the cultivar-dependent HR calculation model will need to be improved. In this study, we developed a modified $\mathrm{BBCH}$ scale useful for examining Japanese apricot floral bud development. Furthermore, we proved that the $\mathrm{BBCH}$ stages are correlated with seasonal changes in bud break competency. Accordingly, $\mathrm{BBCH}$ scales may be useful for characterizing the dormancy (quiescence) status of Japanese apricot floral buds.

Author Contributions: Investigation, T.-F.H. and Y.-J.L.; resources, H.Y. and R.T.; writing-original draft preparation, T.-F.H.; writing-review and editing, H.Y.; supervision, R.T.; project administration, H.Y.; funding acquisition, H.Y. All authors have read and agreed to the published version of the manuscript.

Funding: This research was supported by a Grant-in-Aid for Scientific Research (KAKENHI Nos. $18 \mathrm{H} 02198$ and 21H02186) from the Japan Society for the Promotion of Science.

Acknowledgments: We thank Edanz Group (https: / / en-author-services.edanz.com/ac) (accessed on 7 April 2021) for editing a draft of this manuscript.

Conflicts of Interest: The authors declare no conflict of interest.

\section{References}

1. De Hertogh, A. Principles for forcing tulips, hyacinths, daffodils, easter lilies and dutch irises. Sci. Hortic. 1974, 2, 313-355. [CrossRef]

2. Fadón, E.; Herrero, M.; Rodrigo, J. Dormant flower buds actively accumulate starch over winter in sweet cherry. Front. Plant Sci. 2018, 9, 1-10. [CrossRef]

3. Rohde, A.; Bhalerao, R.P. Plant dormancy in the perennial context. Trends Plant Sci. 2007, 12, 217-223. [CrossRef]

4. Lang, G.A.; Early, J.D.; Martin, G.C.; Darnell, R.L. Endo-, para-, and ecodormancy: Physiological terminology and classification for dormancy research. HortScience 1987, 22, 371-377.

5. Hsiang, T.-F.; Chen, W.; Yamane, H. The MADS-box gene family involved in the regulatory mechanisms of dormancy and flowering in Rosaceae fruit trees. Annu. Plant Rev. Online 2021, in press.

6. Julian, C.; Rodrigo, J.; Herrero, M. Stamen development and winter dormancy in apricot (Prunus armeniaca). Ann. Bot. 2011, 108, 617-625. [CrossRef] [PubMed]

7. Fadón, E.; Herrero, M.; Rodrigo, J. Flower development in sweet cherry framed in the BBCH scale. Sci. Hortic. 2015, 192, 141-147. [CrossRef]

8. Fadón, E.; Herrero, M.; Rodrigo, J. Anther and pollen development in sweet cherry (Prunus avium L.) in relation to winter dormancy. Protoplasma 2019, 256, 733-744. [CrossRef] [PubMed] 
9. Fernandez, E.; Luedeling, E.; Behrend, D.; Van De Vliet, S.; Kunz, A.; Fadón, E. Mild water stress makes apple buds more likely to flower and more responsive to artificial forcing-Impacts of an unusually warm and dry summer in germany. Agronomy 2020, 10, 274. [CrossRef]

10. Penso, G.A.; Citadin, I.; Scariotto, S.; Magalhães dos Santos, C.E.; Junior, A.W.; Bruckner, C.H.; Rodrigo, J. Development of peach flower buds under low winter chilling conditions. Agronomy 2020, 10, 428. [CrossRef]

11. Meier, U. Growth Stages of Mono-and Dicotyledonous Plants; Federal Biological Research Centre for Agriculture and Forestry: Kleinmachnow, Germany, 2001; p. 158.

12. Pérez-Pastor, A.; Ruiz-Sánchez, M.; Domingo, R.; Torrecillas, A. Growth and phenological stages of Búlida apricot trees in south-east Spain. Agronomie 2004, 24, 93-100. [CrossRef]

13. Sakar, E.H.; El Yamani, M.; Boussakouran, A.; Rharrabti, Y. Codification and description of almond (Prunus dulcis) vegetative and reproductive phenology according to the extended BBCH scale. Sci. Hortic. 2019, 247, 224-234. [CrossRef]

14. Fadón, E.; Rodrigo, J. Unveiling winter dormancy through empirical experiments. Environ. Exp. Bot. 2018, 152, 28-36. [CrossRef]

15. Szalay, L.; Froemel-Hajnal, V.; Bakos, J.; Ladányi, M. Changes of the microsporogenesis process and blooming time of three apricot genotypes (Prunus armeniaca L.) in central hungary based on long-term observation (1994-2018). Sci. Hortic. 2019, 246, 279-288. [CrossRef]

16. Fadón, E.; Herrera, S.; Herrero, M.; Rodrigo, J. Male meiosis in sweet cherry is constrained by the chilling and forcing phases of dormancy. Tree Physiol. 2020, 41, 619-630. [CrossRef]

17. McPherson, H.G.; Snelgar, W.P.; Manson, P.J.; Snowball, A.M. Bud respiration and dormancy of kiwifruit (Actinidia deliciosa). Ann. Bot. 1997, 80, 411-418. [CrossRef]

18. Fadón, E.; Herrera, S.; Guerrero, B.I.; Guerra, M.E.; Rodrigo, J. Chilling and heat requirements of temperate stone fruit trees (Prunus sp.). Agronomy 2020, 10, 409. [CrossRef]

19. Weinberger, J.H. Chilling requirements of peach varieties. Proc. Am. Soc. Hortic. Sci. 1950, 56, $122-128$.

20. Richardson, E.A.; Seeley, S.D.; Walker, D.R. A model for estimating the completion of rest for redhaven and elberta peach trees. HortScience 1974, 9, 331-332.

21. Fishman, S.; Erez, A.; Couvillon, G.A. The temperature dependence of dormancy breaking in plants: Mathematical analysis of a two-step model involving a cooperative transition. J. Theor. Biol. 1987, 124, 473-483. [CrossRef]

22. Richardson, E.A.; Seeley, S.D.; Anderson, J.L.; Ashcroft, G.L. Pheno-climatography of spring peach bud development. HortScience 1975, 10, 236-237.

23. Anderson, J.L.; Richardson, E.A. The Utah chill unit/flower bud phenology models for deciduous fruit: Their implication for production in subtropical areas. Acta Hortic. 1987, 199, 45-50. [CrossRef]

24. Götz, K.P.; Chmielewski, F.M.; Homann, T.; Huschek, G.; Matzneller, P.; Rawel, H.M. Seasonal changes of physiological parameters in sweet cherry (Prunus avium L.) buds. Sci. Hortic. 2014, 172, 183-190. [CrossRef]

25. Saito, T.; Tuan, P.A.; Katsumi-Horigane, A.; Bai, S.; Ito, A.; Sekiyama, Y.; Ono, H.; Moriguchi, T. Development of flower buds in the Japanese pear (Pyrus pyrifolia) from late autumn to early spring. Tree Physiol. 2015, 35, 653-662. [CrossRef] [PubMed]

26. Yamamoto, R.R.; Akemi, K.H.; Yoshida, M.; Sekozawa, Y.; Sugaya, S.; Gemma, H. Floral primordia necrosis" Incidence in mixed buds of Japanese pear (Pyrus pyrifolia (Burm.) Nakai var. Culta) 'Housui' grown under mild winter conditions and the possible relation with water dynamics. J. Jpn. Soc. Hortic. Sci. 2010, 79, 246-257.

27. Yamane, H.; Kashiwa, Y.; Kakehi, E.; Yonemori, K.; Mori, H.; Hayashi, K.; Iwamoto, K.; Tao, R.; Kataoka, I. Differential expression of dehydrin in flower buds of two Japanese apricot cultivars requiring different chilling requirements for bud break. Tree Physiol. 2006, 26, 1559-1563. [CrossRef] [PubMed]

28. Kitamura, Y.; Yamane, H.; Yukimori, A.; Shimo, H.; Numaguchi, K.; Tao, R. Blooming date predictions based on Japanese apricot 'Nanko' flower bud responses to temperatures during dormancy. HortScience 2017, 52, 366-370. [CrossRef]

29. Prudencio, A.S.; Martínez-Gómez, P.; Dicenta, F. Evaluation of breaking dormancy, flowering and productivity of extra-late and ultra-late flowering almond cultivars during cold and warm seasons in south-east of Spain. Sci. Hortic. 2018, $235,39-46$. [CrossRef]

30. Okie, W.R.; Blackburn, B. Increasing chilling reduces heat requirement for floral budbreak in peach. HortScience 2011, 46, 245-252. [CrossRef]

31. Heide, O.M. Interaction of photoperiod and temperature in the control of growth and dormancy of Prunus species. Sci. Hortic. 2008, 115, 309-314. [CrossRef]

32. Renaut, J.; Hausman, J.F.; Bassett, C.; Artlip, T.; Cauchie, H.M.; Witters, E.; Wisniewski, M. Quantitative proteomic analysis of short photoperiod and low-temperature responses in bark tissues of peach (Prunus persica L. Batsch). Tree Genet. Genomes 2008, 4, 589-600. [CrossRef]

33. Wisniewski, M.E.; Bassett, C.L.; Renaut, J.; Farrell, R.; Tworkoski, T.; Artlip, T.S. Differential regulation of two dehydrin genes from peach (Prunus persica) by photoperiod, low temperature and water deficit. Tree Physiol. 2006, 26, 575-584. [CrossRef]

34. Vazquez-Hernandez, M.; Romero, I.; Escribano, M.I.; Merodio, C.; Sanchez-Ballesta, M.T. Deciphering the role of CBF/DREB transcription factors and dehydrins in maintaining the quality of table grapes cv. Autumn Royal treated with high $\mathrm{CO}_{2}$ levels and stored at $0{ }^{\circ} \mathrm{C}$. Front. Plant Sci. 2017, 8, 1-15. [CrossRef]

35. Viti, R.; Bartolini, S.; Andreini, L. Apricot flower bud dormancy: Main morphological, anatomical and physiological features related to winter climate influence. Adv. Hortic. Sci. 2013, 27, 5-17. 
36. Julian, C.; Herrero, M.; Rodrigo, J. Anther meiosis time is related to winter cold temperatures in apricot (Prunus armeniaca L.). Environ. Exp. Bot. 2014, 100, 20-25. [CrossRef]

37. Sasaki, R.; Yamane, H.; Ooka, T.; Jotatsu, H.; Kitamura, Y.; Akagi, T.; Tao, R. Functional and expressional analyses of PmDAM genes associated with endodormancy in Japanese apricot. Plant Physiol. 2011, 157, 485-497. [CrossRef]

38. Considine, M.J.; Considine, J.A. On the language and physiology of dormancy and quiescence in plants. J. Exp. Bot. 2016, 67, 3189-3203. [CrossRef] 